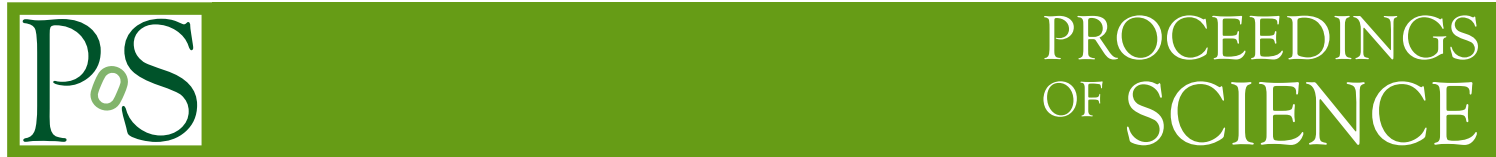

\title{
Theory of Kaon Physics
}

\section{Giancarlo D’Ambrosio*i}

Theory Division, Physics Department, CERN, CH-1211 Geneva 23, Switzerland

Istituto Nazionale di Fisica Nucleare, Sezione di Napoli, Complesso Universitario di Monte S.

Angelo, Via Cintia Edificio 6, 80126 Napoli, Italy

E-mail: gdambros@na.infn.it

I review rare kaon decays. I introduce the flavor problem and possible solutions. Very rare kaon decays like $K \rightarrow \pi v \bar{v}$ are very important to this purpose but also $K \rightarrow \pi l^{+} l^{-}$. A new interesting channel is $K \rightarrow \pi \pi e e$. Chiral dynamics is important to disentangle short distance effects. We discuss also the decays $K^{0} \rightarrow \mu^{+} \mu^{-}$, which have received recently some attention due to the measurement by LHCB.therefore we will study also $K_{L} \rightarrow \pi^{0} e^{+} e^{-}, K^{+} \rightarrow \pi^{+} \pi^{0} \gamma, K^{+} \rightarrow$ $\pi^{+} \pi^{0} e^{+} e^{-}$and related channels

XIIth International Conference on Heavy Quarks \& Leptons 2014,

25-29 August 2014

Schloss Waldthausen, Mainz, Germany

\footnotetext{
* Speaker.

${ }^{\dagger}$ This work was partially supported by MIUR under project 2010YJ2NYW.
} 


\section{Introduction}

I am honoured to give a discussion on rare kaon decays in Nagoya University where many footprints of the Standard Model have been left has also by Kobayashi's talk; indeed a good start of the flavour story would start discussing Cabibbo and Kobayashi-Maskawa's papers (see Kobayashi's talk and [1]), GIM paper but also, according to me, Gaillard-Lee's [2] and Inami Lin papers [3]: with the first paper uses the two family SM to compute rare kaon decays basically computing with phenomenological meson models, i.e. pre-CHPT, and neglecting strong interaction effects in short distance diagrams; the second paper adds the third family and this oblige to study an interesting and important limit $m_{t}$ heavy A systematic approach to evaluate the relevant QCD correction in the OPE expansion has been successful developed by Buras and collaborators [4] QCD correction program (see for instance refs in [5]) and phenomenological determination of the CKM parameters have married nicely for instance to determine that New Physics (NP) corrections to the $\Delta F=2$ GIM formula for

$$
\mathscr{H}_{\Delta F=2}^{S M} \sim \frac{G_{F}^{2} M_{W}^{2}}{16 \pi^{2}}\left[\frac{\left(V_{t d}^{*} m_{t}^{2} V_{t b}\right)^{2}}{\mathrm{v}^{4}}\left(\bar{d}_{L} \gamma^{\mu} b_{L}\right)^{2}+\frac{\left(V_{t d}^{*} m_{t}^{2} V_{t s}\right)^{2}}{\mathrm{v}^{4}}\left(\bar{d}_{L} \gamma^{\mu} s_{L}\right)^{2}\right]+\text { charm }
$$

are very constrained; here Inami - Lin functions have been written as polynomials and retained only the leading term, then QCD can be applied and then one

Flavour physics is also important to address properly extensions of the SM; generic new flavor structures are strongly constrained pushing the new physics scale to a very large value $(\sim 100$ $\mathrm{TeV}$ ) creating tension with naturalness. An interesting global symmetry, minimal flavour violation (MFV), was introduced to avoid large FCNC; the SM lagrangian has an interesting symmetry in the limit that all the fermionic sector is massless: defining $Q$ 's, $U$ 's and $D$ 's, the left-handed doublets, right-handed up singlets and right-handed down singlets, the global symmetry, $\mathscr{G}_{F}=$ $U(3)_{Q} \times U(3)_{U} \times U(3)_{D}$, is conserved. This global symmetry is broken by the mass terms, i.e. the Yukawas. These Yukawas must be the only sources of the flavour group, $\mathscr{G}_{F}$, breaking so that then the effective FCNC hamiltonian is

$$
\mathscr{H}_{\Delta F=2}^{S M} \sim \frac{G_{F}^{2} M_{W}^{2}}{16 \pi^{2}}\left[\frac{\left(V_{t d}^{*} m_{t}^{2} V_{t b}\right)^{2}}{v^{4}}\left(\bar{d}_{L} \gamma^{\mu} b_{L}\right)^{2}+\frac{\left(V_{t d}^{*} m_{t}^{2} V_{t s}\right)^{2}}{v^{4}}\left(\bar{d}_{L} \gamma^{\mu} s_{L}\right)^{2}\right]+\text { charm }
$$

One then requires that New Physics does not add any new flavour structures: NP have the same SM flavor breaking, i.e. the Yukawas leading to an effective hamiltonian proportional to eq.(1.2). This effective approach to flavour physics beyond the Standard Model is the so called minimal flavor violation (MFV) $[7,8,9,10]$.

\section{THE ULTRA-RARE DECAY $K^{+} \rightarrow \pi^{+} v \bar{v}$}

Rare kaon decays furnish challenging MFV probes and will severely constrain additional flavor physics motivated by NP. SM predicts the $V-A \otimes V-A$ effective hamiltonian

$$
\mathscr{H}=\frac{G_{F}}{\sqrt{2}} \frac{\alpha}{2 \pi \sin ^{2} \theta_{W}}(\underbrace{V_{c s}^{*} V_{c d} X_{N L}}_{\lambda x_{c}}+\underbrace{V_{t s}^{*} V_{t d} X\left(x_{t}\right)}_{A^{2} \lambda^{5}(1-\rho-i \eta) x_{t}}) \bar{s}_{L} \gamma_{\mu} d_{L} \bar{v}_{L} \gamma^{\mu} v_{L},
$$


$x_{q}=m_{q}^{2} / M_{W}^{2}, \theta_{W}$ the Weak angle and $X$ 's are the Inami-Lin functions with Wilson coefficients known at two-loop electroweak corrections [12]. $S U(2)$ isospin symmetry relates hadronic matrix elements for $K \rightarrow \pi v \bar{v}$ to $K \rightarrow \pi l \bar{v}$ to a very good precision [16] while long distance contributions and QCD corrections are under control [12] and the main uncertainties is due to the strong corrections in the charm loop contribution. The structure in (2.1) leads to a pure CP violating contribution to $K_{L} \rightarrow \pi^{0} v \bar{v}$, induced only from the top loop contribution and thus proportional to $\mathfrak{I} m\left(\lambda_{t}\right)\left(\lambda_{t}=V_{t s}^{*} V_{t d}\right)$ and free of hadronic uncertainties. This leads to the prediction [12]

$$
\mathscr{B}\left(K^{ \pm}\right)_{S M}=(8.22 \pm 0.69 \pm 0.29) \times 10^{-11} \quad \mathscr{B}\left(K_{L}\right)_{S M}=\left(2.43_{-0.37}^{+0.40}+0.06\right) \times 10^{-11}
$$

where the first is the parametric uncertainty due to the error on $\left|V_{c b}\right|, \rho$ and $\eta, f_{K}$, and the second error summarises the theoretical uncertainties on non-perturbative physics and QCD higher order terms.

It is also possible to be more explicit on the CKM-dependence, by using the central PDG value for and then writing [13]

$$
\begin{gathered}
\mathscr{B}\left(K^{ \pm}\right)_{S M}=(8.39 \pm 0.30) \times 10^{-11}\left[\frac{\left|V_{c b}\right|}{40.7 \times 10^{-3}}\right]^{2.8}\left[\frac{\gamma}{73.2^{\circ}}\right]^{0.708} \\
\mathscr{B}\left(K_{L}\right)_{S M}=(3.36 \pm 0.05) \times 10^{-11}\left[\frac{\left|V_{u b}\right|}{3.88 \times 10^{-3}}\right]^{2}\left[\frac{\left|V_{c b}\right|}{40.7 \times 10^{-3}}\right]^{2}\left[\frac{\sin \gamma}{\sin \left(73.2^{\circ}\right)}\right]^{2}
\end{gathered}
$$

$K^{ \pm} \rightarrow \pi^{ \pm} v \bar{v}$ receives CP conserving contributions proportional to $\Re e\left(\lambda_{c}\right)$, and to $\Re e\left(\lambda_{t}\right)$ and a CP violating one proportional to $\mathfrak{I} m\left(\lambda_{t}\right)$. E949 Collaboration [17] and E391a Collaboration [18] have then measured

$$
\begin{gathered}
\mathscr{B}\left(K^{ \pm}\right)=\left(1.73_{-1.05}^{+1.15}\right) \times 10^{-10} \quad \text { E949 } \\
\mathscr{B}\left(K_{L}\right)<2.6 \times 10^{-8} \text { at } 90 \% \text { C.L. E391aCollaboration }
\end{gathered}
$$

The direct upper bound for the neutral decay can be improved with a theoretical analysis: the isospin structure of any $\bar{s} d$ operator (bilinear in the quark fields) leads to the model independent relation among $A\left(K_{L} \rightarrow \pi^{0} v \bar{v}\right)$ and $A\left(K^{ \pm} \rightarrow \pi^{ \pm} v \bar{v}\right)$ [19]; this leads to

$$
\mathscr{B}\left(K_{L} \rightarrow \pi^{0} v \bar{v}\right)<4 \mathscr{B}\left(K^{ \pm} \rightarrow \pi^{ \pm} v \bar{v}\right)
$$

The upcoming KOTO experiment [16, 20] for $K_{L} \rightarrow \pi^{0} v \bar{v}$, NA62 [21] for $\left(K^{ \pm} \rightarrow \pi^{ \pm} v \bar{v}\right)$ encourage theoretical investigations of extensions of the SM: these experiments probe deeply to the MFV scale [10]. More aggressive NP models can furnish substantial enhancements and be either discovered or ruled out $[12,22]$ !

\section{3. $K_{L} \rightarrow \pi^{0} l l$ and $K_{S} \rightarrow \pi^{0} e^{+} e^{-}$}

The electroweak short distance contribution to $K_{L} \rightarrow \pi^{0} e^{+} e^{-}$, analogously to $K_{L} \rightarrow \pi^{0} v \bar{v}$, violates directly $\mathrm{CP}$ violation; however there is long distance contamination due to electromagnetic interactions: i) a negligible 
CP conserving contribution due to two-photon exchange, (from the measured $K_{L} \rightarrow \pi^{0} \gamma \gamma$ spectrum $\mathscr{B}\left(K_{L} \rightarrow \pi^{0} e^{+} e^{-}\right)_{\gamma \gamma}<5 \cdot 10^{-13}$ at $\left.90 \% \quad \mathrm{CL}\right)$ and ii) an indirect CP violating contribution mediated by one photon exchange, i.e. the contribution suppressed by $\varepsilon$ in $K_{L} \sim K_{2}+\varepsilon K_{1} \rightarrow$ $\pi^{0} e^{+} e^{-}$determined by the CP conserving $A\left(K_{S} \rightarrow \pi^{0} e^{+} e^{-}\right)[23,24]$.

The CP-conserving decays $K^{ \pm}\left(K_{S}\right) \rightarrow \pi^{0} \ell^{+} \ell^{-}$are dominated by the long-distance process $K \rightarrow \pi \gamma^{*} \rightarrow \pi \ell^{+} \ell^{-}$[24]. Our ignorance in the long distance dominated $A\left(K_{S} \rightarrow \pi^{0} l^{+} l^{-}\right)$can be parametrized by one parameter $a_{S}$ to be determined experimentally. NA48, finds in the electron $\left|a_{S}\right|_{e e}=1.06_{-0.21}^{+0.26} \pm 0.07$ and muon final state $\left|a_{S}\right|_{\mu \mu}=1.54_{-0.32}^{+0.40} \pm 0.06$ These results allow us to evaluate the $\mathrm{CP}$ violating branching

$$
B\left(K_{L} \rightarrow \pi^{0} e^{+} e^{-}\right)_{C P V}=\left[15.3 a_{S}^{2}-6.8 \frac{\mathfrak{I} \lambda_{t}}{10^{-4}} a_{S}+2.8\left(\frac{\mathfrak{I} \lambda_{t}}{10^{-4}}\right)^{2}\right] \times 10^{-12},
$$

The first term is the indirect $\mathrm{CP}$ violating contribution while the last term is the direct $\mathrm{CP}$ violating contribution; the second one is the interference, expected constructive. This allows a stronger signal [23]. This prediction is not far from the the present bound from $\mathrm{KTeV}$ [25],

$$
\mathscr{B}\left(K_{L} \rightarrow \pi^{0} e^{+} e^{-}\right)<2.8 \times 10^{-10} \text { at } 90 \% \quad \mathrm{CL} .
$$

which also sets the interesting limit $\mathscr{B}\left(K_{L} \rightarrow \pi^{0} \mu^{+} \mu^{-}\right)<3.8 \times 10^{-10}$ [25],

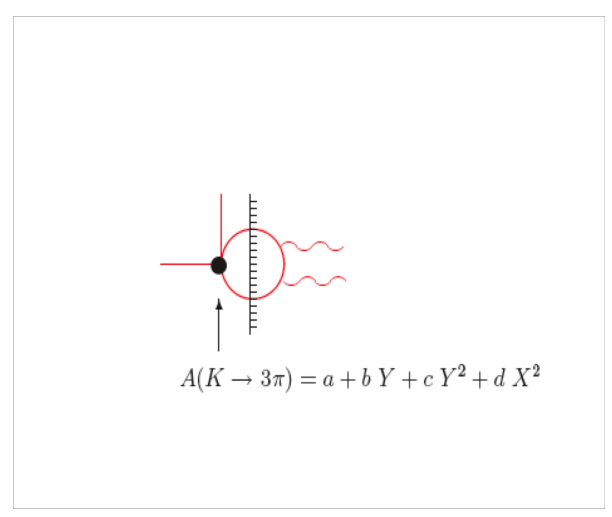

Figure 1: Unitarity contributions to $K \rightarrow \pi \gamma \gamma$

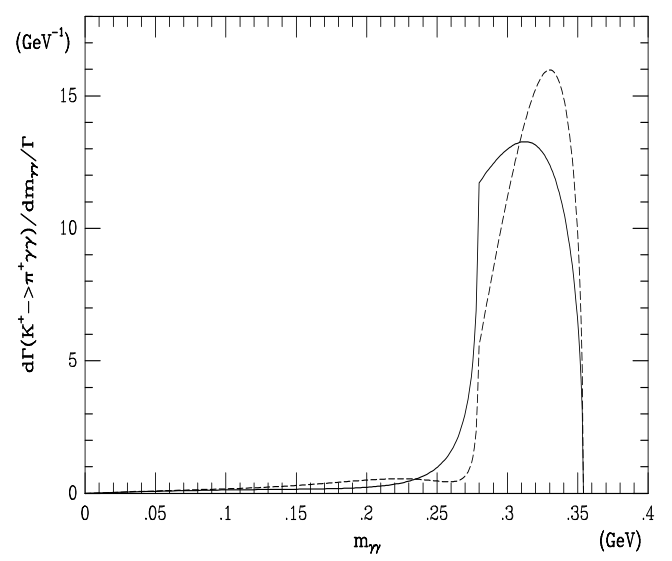

Figure 2: $K^{+} \rightarrow \pi^{+} \gamma \gamma: \hat{c}=0$, full line, $\hat{c}=$ -2.3 , dashed line, [26]

Recently a related channel, $K^{+} \rightarrow \pi^{+} \gamma \gamma$, has attracted attention: new measurements of this decay have been performed using minimum bias data sets collected during a 3-day special NA48/2 run in 2004 with $60 \mathrm{GeV} K^{ \pm}$beams, and a 3-month NA62 run in 2007 with $74 \mathrm{GeV} / \mathrm{c} K^{ \pm}$beams [21].

This channel starts at $\mathscr{O}\left(p^{4}\right)$, with pion (and kaon) loops and a local term $\hat{c}$. Due to the presence of the pion pole, there is a new helicity amplitude, $C$ [24]; the unitarity contributions at $\mathscr{O}\left(p^{6}\right)$ in Fig. 1 enhance the amplitude $A$ by $30 \%-40 \%$, along with the generation of a $B$-type amplitude [26]; as a result the differential decay rate is

$$
\frac{d^{2} \Gamma}{d y d z} \sim\left[z^{2}\left(|A+B|^{2}+|C|^{2}\right)+\left(y^{2}-\left(\frac{\left(1+r_{\pi}^{2}-z\right)^{2}}{4}-r_{\pi}^{2}\right)\right)^{2}|B|^{2}\right]
$$


The constant $\hat{c}$ can be fixed by a precise determination of the rate and the spectrum as shown in Fig.2 [26]; this constant is predicted in terms of strong and weak counterterms, generated from the axial spin- 1 contributions

$$
\left.\hat{c}=\frac{128 \pi^{2}}{3}\left[3\left(L_{9}+L_{10}\right)+N_{14}-N_{15}-2 N_{18}\right)\right] \stackrel{\mathrm{FM}}{=} 2.3\left(1-2 k_{f}\right),
$$

with $k_{f}$ is the factorization factor in the factorization model (FM) model [27]. BNL 787 got 31 events leading to $B\left(K^{+} \rightarrow \pi^{+} \gamma \gamma\right) \sim(6 \pm 1.6) \cdot 10^{-7}$ and a value of $\hat{c}=1.8 \pm 0.6$ [25]. New measurements of this decay have been performed using data collected during a 3-day special NA48/2 run in 2004 and a 3-month NA62 run in 2007. Signal events are selected in the region of $z=m_{\gamma \gamma}^{2} / m_{K}^{2}>0.2$ leading to $\mathscr{B}\left(K^{+} \rightarrow \pi^{+} \gamma \gamma\right)=(1.003 \pm 0.056) \cdot 10^{-6}$ and $\hat{c}=1.87 \pm 0.24_{\text {stat }} \pm 0.09_{\text {syst }}[21,28,29]$.

\section{4. $K \rightarrow \pi \pi \gamma$ and $K \rightarrow \pi \pi e e$-decays}

$\mathrm{CP}$ violation has been also studied in the $K \rightarrow \pi \pi \gamma$ and $K \rightarrow \pi \pi e e$ decays. According to gauge and Lorentz invariance we decompose $K(p) \rightarrow \pi\left(p_{1}\right) \pi\left(p_{2}\right) \gamma(q)$ decays, in electric $(E)$ and magnetic $(M)$ amplitudes [30]. Particularly interesting are the recent interesting NA48/2 data regarding $K^{+} \rightarrow \pi^{+} \pi^{0} \gamma$ decays [31]. Due to the $\Delta I=3 / 2$ suppression of the bremsstrahlung, interference between $E_{B}$ and the electric dipole $(E 1)$ and magnetic transitions can be measured. Defining $z_{i}=p_{i} \cdot q / m_{K}^{2} \quad z_{3}=p_{K} \cdot q / m_{K}^{2}$ and $z_{3} z_{+}=\frac{m_{\pi^{+}}^{2}}{m_{K}^{2}} W^{2}$ we can study the deviation from bremsstrahlung

$$
\frac{\partial^{2} \Gamma}{\partial T_{c}^{*} \partial W^{2}}=\frac{\partial^{2} \Gamma_{I B}}{\partial T_{c}^{*} \partial W^{2}}\left[1+\frac{m_{\pi^{+}}^{2}}{m_{K}} 2 \operatorname{Re}\left(\frac{E_{D E}}{e A}\right) W^{2}+\frac{m_{\pi^{+}}^{4}}{m_{K}^{2}}\left(\left|\frac{E_{D E}}{e A}\right|^{2}+\left|\frac{M_{D E}}{e A}\right|^{2}\right) W^{4}\right],
$$

where $A=A\left(K^{+} \rightarrow \pi^{+} \pi^{0}\right)$. Study of the Dalitz plot has lead NA48 to the results [31]

Table 3

\begin{tabular}{lc} 
NA48/2 & $T_{c}^{*} \in[0,80] \mathrm{MeV}$ \\
\hline $\operatorname{Frac}(D E)=(3.32 \pm 0.15 \pm 0.14) \times 10^{-2}$ \\
$\operatorname{Frac}(I N T)=(-2.35 \pm 0.35 \pm 0.39) \times 10^{-2}$
\end{tabular}

Also the interesting CP bound was obtained [31]:

$$
\frac{\Gamma\left(K^{+} \rightarrow \pi^{+} \pi^{0} \gamma\right)-\Gamma\left(K^{-} \rightarrow \pi^{-} \pi^{0} \gamma\right)}{\Gamma\left(K^{+} \rightarrow \pi^{+} \pi^{0} \gamma\right)+\Gamma\left(K^{-} \rightarrow \pi^{-} \pi^{0} \gamma\right)}<1.5 \cdot 10^{-3} \quad \text { at } 90 \% \quad \text { CL. }
$$

We have studied the decay $K^{ \pm} \rightarrow \pi^{ \pm} \pi^{0} e^{+} e^{-}$, dominated by long distance through one photon exchange in Fig. 3. We computed the large long distance contributions and the relatively small short distance ones in Ref. [32, 33], see in Fig.5 the size of the different LD contributions. Relatively to the $K^{ \pm} \rightarrow \pi^{ \pm} \pi^{0} \gamma$ the possibility to go kinematically at large $q^{2}$ opens the possibility to beat the bremsstrahlung (Fig.5): at large dilepton invariant mass the bremsstrahlung can be even 100 time smaller than the magnetic contribution, however at the price of decreasing the statistics. The further advantage is that at each $q^{2}$ we can measure the different Dalitz plot, as we can see in Fig. 4, obtained at $\sqrt{q^{2}}=50 \mathrm{MeV}$. Also several short distance observables (Fig.6) can be measured by appropriate kinematical analyses [32,33]. The big news is that NA62 has reported a measurement 
of this branching, the experiment selects 3 reconstructed tracks coming from one decay vertex, then Particle ID for $e^{ \pm} / \pi^{ \pm}$separation, then two reconstructed $\gamma$ clusters compatible with $\pi^{0}$ mass [34] The number of $K^{ \pm}$decays (kaon flux) is measured by using the reference channel $K^{ \pm} \rightarrow \pi^{ \pm} \pi^{0}(\gamma)$ : 1860 genuine $K^{+} \rightarrow \pi^{+} \pi^{0} e^{+} e^{-}$events are selected after background subtraction or a branching ratio $\left(4.06 \pm 0.10_{\text {stat. }} \pm 0.06_{\text {syst. }} \pm 0.13_{\text {ext. }}\right) \times 10^{-6}$ the external error comes from Dalitz decays $\pi^{0} \rightarrow e^{+} e^{-} \gamma$

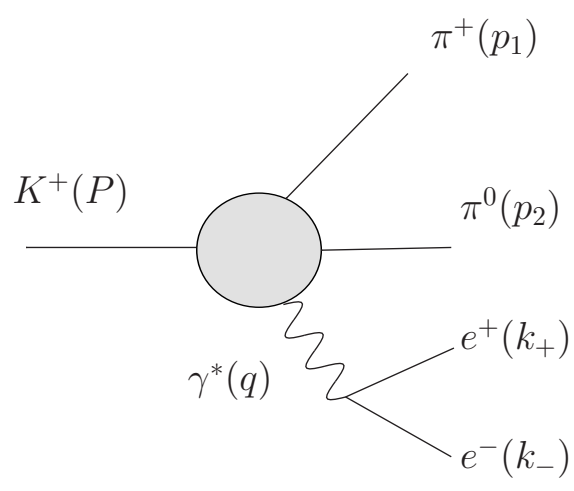

Figure 3: $K^{ \pm} \rightarrow \pi^{ \pm} \pi^{0} e^{+} e^{-}$: long distance contributions dominated by one photon exchange

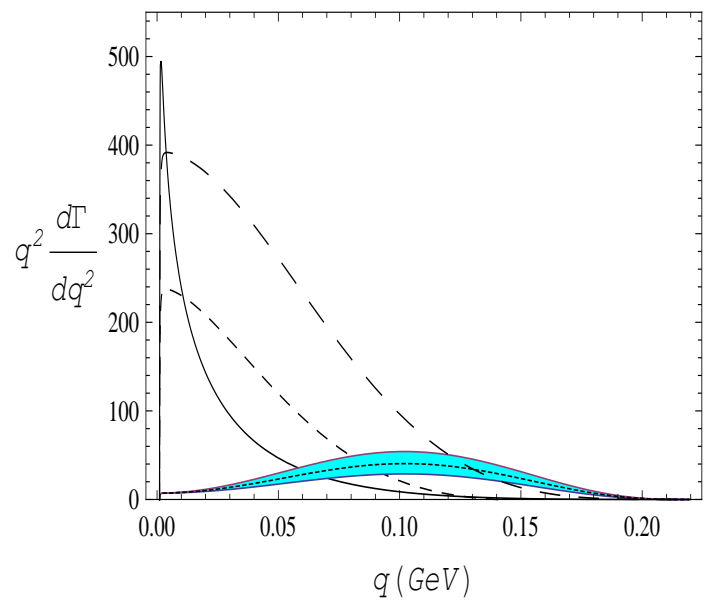

Figure 5: $q$ dependence of Bremsstrahlung (solid line), the dashed lines (from bigger to smaller) are $100 \times M, 100 \times B E$ and $300 \times$ E. Error bars are omitted. The band corresponds to changing counterterms.

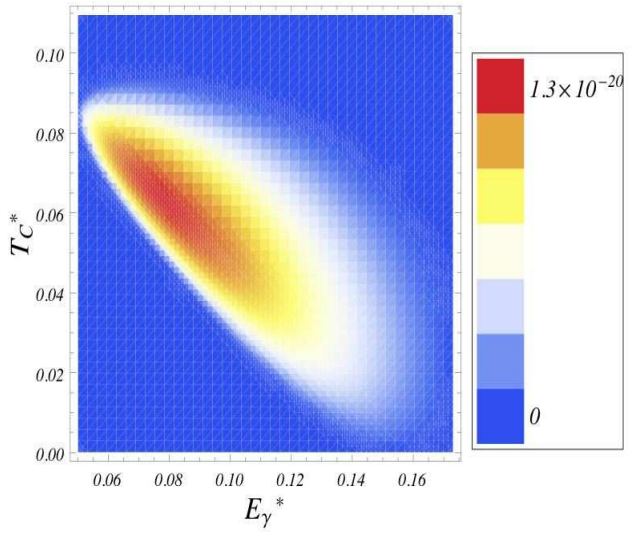

Figure 4: Dalitz plot BM contribution: twodimensional density projection

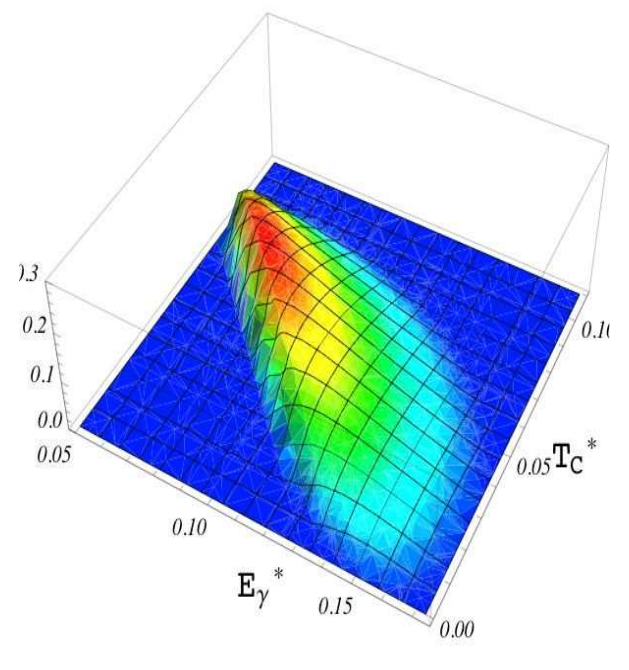

Figure 6: Dalitz plot in the $\left(E_{\gamma}^{*}, T_{c}^{*}\right)$ plane at $q^{2}=$ $(50 \mathrm{MeV})^{2}$ for the P-violating BM contribution

A good way to detect short distance physics in $K_{L, S} \rightarrow \pi^{+} \pi^{-} l^{+} l^{-}$or $K^{ \pm} \rightarrow \pi^{ \pm} \pi^{0} l^{+} l^{-}$is to measure the interference with axial leptonic current (see refs. in [32]): various diplane angular 
Table 1: Results for the $K_{S}$ decays. Notice that there are no experimental results.

\begin{tabular}{c|c|c|c|} 
& $\alpha_{S}=\beta_{S}=0$ & $\alpha_{S}=0, \beta_{S}=-1$ & $\alpha_{S}=\alpha_{L}$ \\
\hline$K_{S} \rightarrow \mu \bar{\mu} \mu \bar{\mu}$ & $1.40 \times 10^{-14}$ & $1.37 \times 10^{-14}$ & $2.61 \times 10^{-14}$ \\
$K_{S} \rightarrow e \bar{e} e \bar{e}$ & $1.66 \times 10^{-10}$ & $1.66 \times 10^{-10}$ & $1.78 \times 10^{-10}$ \\
$K_{S} \rightarrow \mu \bar{\mu} e \bar{e}$ & $7.88 \times 10^{-12}$ & $7.87 \times 10^{-12}$ & $1.29 \times 10^{-11}$ \\
\hline
\end{tabular}

Table 2: Results for the $K_{L}$ decays

\begin{tabular}{ccc}
\hline$\alpha_{L}=\beta_{L}=0$ & $\alpha_{L}=-1.63$ & Experiment \\
\hline This work & This Work & PDG [25] \\
$4.82 \times 10^{-13}$ & $8.78 \times 10^{-13}$ & - \\
$3.40 \times 10^{-8}$ & $3.65 \times 10^{-8}$ & $(3.56 \pm 0.21) \times 10^{-8}$ \\
$1.55 \times 10^{-9}$ & $2.51 \times 10^{-9}$ & $(2.69 \pm 0.27) \times 10^{-9}$ \\
\hline
\end{tabular}

asymmetries have been studied to this purpose [32] and this possibility possibly opens new directions to disentangle short distance from long distance.

\section{5. $K_{S, L} \rightarrow l^{+} l^{-}, K_{S, L} \rightarrow l^{+} l^{-} l^{+} l^{-}[25,35,38]$}

Recent LHCB limit on $K_{S} \rightarrow \mu \bar{\mu}$ [25] in the table is close to test interesting New Physics (NP) models [35]. A high precision measurement can test the short distance (SD) SM but it requires to improve the long distance (LD) prediction [35, 36, 37] with auxiliary channels [38]. $K_{L} \rightarrow \mu \mu$ : the small ratio $\mathrm{SD} / \mathrm{LD} \sim \frac{1}{30}$ may obscure an experimental improvement on the rate [35]. The situation would be a bit ameliorated if the sign for theoretical unknown sign of $A\left(K_{L} \rightarrow \gamma \gamma\right)$ would be known.

This ambiguity, unknown sign of $A\left(K_{L} \rightarrow \gamma \gamma\right)$, could come from the experimental study of $K_{S, L} \rightarrow l^{+} l^{-} l^{+} l^{-}$[38]: see Tables 1 and 2, Figs.7. As shown in table these channels are at reach in a high intensity machine and they may also give LD distance info needed for a better control of $K_{L} \rightarrow \mu \mu$. These four body decays have also a peculiar feature, similarly to $K_{S, L} \rightarrow \pi^{+} \pi^{-} e^{+} e^{-}$, the two different helicity amplitudes interfere; then one can measure the sign $K_{L} \rightarrow \rightarrow \gamma^{*} \gamma^{*} \rightarrow l^{+} l^{-} l^{+} l^{-}$ by studying the time interference $K_{S} K_{L}$ which it has a decay length $2 \Gamma_{S}$ [38].

\section{Conclusions}

We are looking forward to the upcoming $K_{L} \rightarrow \pi^{0} v \bar{v}$ KOTO $[16,20]$ and $K^{+} \rightarrow \pi^{+} v \bar{v}$ [21] NA62 experiments probing deeply the flavour structure of the SM. We have also shown that there are other decay modes like $K_{L} \rightarrow \pi^{0} e^{+} e^{-}, K^{+} \rightarrow \pi^{+} \gamma \gamma$ and $K^{+} \rightarrow \pi^{+} \pi^{0} e^{+} e^{-}$which are very 

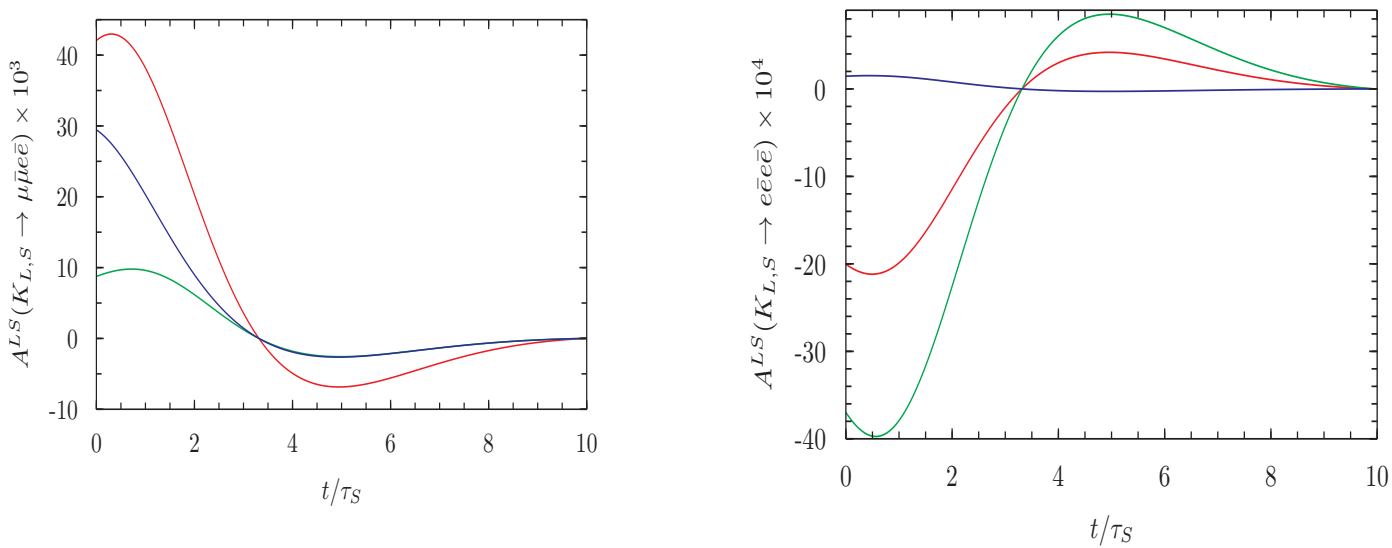

Figure 7: Interferences between $K_{L}$ and $K_{S} \rightarrow \ell_{1} \bar{\ell}_{1} \ell_{2} \bar{\ell}_{2}$. The red line corresponds to the case $\alpha_{S}=0$, the green line is $\alpha_{S}=-3$ while the blue line is $\alpha_{S}=3$. As explained in the text we assume the sign $K_{L} \rightarrow \gamma \gamma$. The interferences being directly related to this sign, their experimental observations (in shape and amplitude) could confirmed this hypothesis.

useful, in particular these last two have been studied recently by NA62. Below we show several interesting channels

\section{Channels interesting in the near future}

$$
\begin{aligned}
& \text { PDG Prospects } \\
& K_{S} \rightarrow \mu \mu \quad<9 \times 10^{-9} \text { at } 90 \% \text { CL }(\mathrm{LD})(5.0 \pm 1.5) \cdot 10^{-12} \quad \mathrm{NP}<10^{-11} \\
& K_{L} \rightarrow \mu \mu \quad(6.84 \pm 0.11) \times 10^{-9} \quad \text { difficult : SD }<<\mathrm{LD} \\
& \begin{array}{lll}
K_{S} \rightarrow \mu \mu \mu \mu & - & \text { SM LD } \sim 2 \times 10^{-14}
\end{array} \\
& K_{S} \rightarrow e e \mu \mu \sim \quad-\quad \sim 10^{-11} \\
& K_{S} \rightarrow \text { eеeе } \quad-\quad \sim 10^{-10} \\
& \begin{array}{lll}
K_{S} \rightarrow \pi^{+} \pi^{-} \mu^{+} \mu^{-} & - & \text {SM LD } \sim 10^{-14}
\end{array}
\end{aligned}
$$

I would like also to mention CPT tests in kaon decays [39] through Bell-Steinberger relations, recently updated in [25]; these leads to best CPT limit and an accurate determination of the $\mathrm{CP}$ violating parameter $\varepsilon$.

\section{Acknowledgements}

Work supported in part by MIUR under project 2010YJ2NYW and by INFN research initiative PhenoLNF. I thank the organizers of HQL2014.

\section{References}

[1] E. de Rafael, in CP Violation and the limits of the Standard Model, TASI 1994 proceedings, ed. J.F. Donoghue (World Scientific, 1995), [hep-ph/9502254]; A. Pich, Rept. Prog. Phys. 58 (1995) 563, [hep-ph/9502336]; G. Ecker, Prog. Part. Nucl. Phys. 35 (1995) 1, [hep-ph/9501357]; G. D'Ambrosio and G. Isidori, Int. J. Mod. Phys. A 13 (1998) 1 [hep-ph/9611284] G. Colangelo and G. Isidori, [hep-ph/0101264]. 
[2] M. K. Gaillard and B. W. Lee, Phys. Rev. D 10, 897 (1974).

[3] T. Inami and C. S. Lim, Prog. Theor. Phys. 65, 297 (1981) [Prog. Theor. Phys. 65, 1772 (1981)].

[4] G. Buchalla, A. J. Buras and M. E. Lautenbacher, Rev. Mod. Phys. 68, 1125 (1996) [hep-ph/9512380].

[5] A. J. Buras, D. Buttazzo, J. Girrbach-Noe and R. Knegjens, arXiv:1503.02693 [hep-ph];

[6] R. Knegjens, arXiv:1505.04928 [hep-ph].

[7] R. S. Chivukula, and H. Georgi, Phys.Lett. B188, 99 (1987).

[8] L. Hall, and L. Randall, Phys.Rev.Lett. 65, 2939-2942 (1990).

[9] A. Buras, et al., Phys.Lett. B500, 161-167 (2001), [hep-ph/0007085].

[10] G. D’ Ambrosio, G. F. Giudice, G. Isidori and A. Strumia, Nucl.Phys. B 645 (2002) 155, [hep-ph/0207036].

[11] K. A. Olive et al. [Particle Data Group Collaboration], Chin. Phys. C 38, 090001 (2014).

[12] M. Gorbahn, M. Patel and S. Robertson, arXiv:1104.0826 [hep-ph]: J. Brod, M. Gorbahn and E. Stamou, Phys. Rev. D83, 034030 (2011); M. Antonelli, D. M. Asner, D. A. Bauer, T. G. Becher, M. Beneke, A. J. Bevan, M. Blanke and C. Bloise et al., Phys. Rept. 494, 197 (2010) [arXiv:0907.5386 [hep-ph]].

[13] A. J. Buras, D. Buttazzo and R. Knegjens, arXiv:1507.08672 [hep-ph],

[14] R. Knegjens, arXiv:1505.04928 [hep-ph].

[15] A. Pich, arXiv:1208.2134 [hep-ph].

[16] T. K. Komatsubara, Prog. Part. Nucl. Phys. 67, 995 (2012) [arXiv:1203.6437 [hep-ex]].

[17] A. V. Artamonov et al. [E949 Collaboration], Phys. Rev. Lett. 101, 191802 (2008) [arXiv:0808.2459 [hep-ex]].

[18] J. K. Ahn et al. [E391a Collaboration], Phys. Rev. D 81, 072004 (2010) [arXiv:0911.4789 [hep-ex]].

[19] Y. Grossman and Y. Nir, Phys. Lett. B 398, 163 (1997) [hep-ph/9701313].

[20] http://koto.kek.jp/

[21] E. Goudzovski, arXiv:1112.5365 [hep-ex]; M. S. Sozzi, arXiv:1102.0893 [hep-ex].

[22] D. M. Straub, arXiv:1012.3893 [hep-ph]; G. Isidori, F. Mescia, P. Paradisi, C. Smith and S. Trine, JHEP 0608, 064 (2006) [hep-ph/0604074].

[23] G. Buchalla, G. D’Ambrosio and G. Isidori, Nucl. Phys. B 672, 387 (2003) [hep-ph/0308008].

[24] G. Ecker, A. Pich and E. de Rafael, Nucl. Phys. B 291, 692 (1987); G. Ecker, A. Pich and E. de Rafael, Nucl. Phys. B 303, 665 (1988); G. D’Ambrosio, G. Ecker, G. Isidori and J. Portolés, JHEP 08, 004 (1998).

[25] K. A. Olive et al. [Particle Data Group Collaboration], Chin. Phys. C 38, 090001 (2014).

[26] G. D’Ambrosio and J. Portoles, Phys. Lett. B 386, 403 (1996) [Phys. Lett. B 389, 770 (1996 ERRAT,B395,390.1997)] [arXiv:hep-ph/9606213].

[27] G. Ecker, J. Kambor and D. Wyler, Nucl. Phys. B 394, 101 (1993); G. D’Ambrosio and J. Portoles, Nucl. Phys. B 533 (1998) 494 [hep-ph/9711211]; L. Cappiello, O. Cata and G. D’Ambrosio, Phys. Rev. D 85, 015003 (2012) [arXiv:1106.0467 [hep-ph]]. 
[28] J. R. Batley et al. [NA48/2 Collaboration], Phys. Lett. B 730, 141 (2014) [arXiv:1310.5499 [hep-ex]].

[29] C. Lazzeroni et al. [NA62 Collaboration], Phys. Lett. B 732, 65 (2014) [arXiv:1402.4334 [hep-ex]].

[30] L. Cappiello and G. D’Ambrosio, Phys. Rev. D 75, 094014 (2007) [arXiv:hep-ph/0702292].

[31] J. R. Batley et al. [NA48/2 Collaboration], Eur. Phys. J. C 68, 75 (2010) [arXiv:1004.0494 [hep-ex]].

[32] L. Cappiello, O. Cata, G. D’Ambrosio and D. -N. Gao, Eur. Phys. J. C 72, 1872 (2012) [arXiv:1112.5184 [hep-ph]].

[33] S. R. Gevorkyan and M. H. Misheva, Eur. Phys. J. C 74, no. 5, 2860 (2014) [arXiv:1403.1053 [hep-ph]].

[34] Mauro Raggi On behalf of the NA48/2 collaboration "NA48 Studies of Kaon Rare Decays "at Les Rencontres de Physique de la Vallée d?Aoste, La Thuile, Aosta Valley, Italy March 1-7, 2015; Milena Misheva on behalf of the NA48/2 Collaboration "First observation and study of the rare decay $K^{ \pm} \rightarrow \pi^{ \pm} \pi^{0} e^{+} e^{-}$by the NA48/2 experiment "at Rencontres de Moriond QCD and High Energy Interactions 2015

[35] G. Isidori and R. Unterdorfer, JHEP 0401, 009 (2004) [hep-ph/0311084].

[36] G. Ecker and A. Pich, Nucl. Phys. B 366, 189 (1991);

[37] G. D’Ambrosio, G. Isidori and J. Portolés, Phys. Lett. B 423, 385 (1998).

[38] G. D’Ambrosio, D. Greynat and G. Vulvert, Eur. Phys. J. C 73, 2678 (2013) [arXiv:1309.5736 [hep-ph]].

[39] F. Ambrosino et al. [KLOE Collaboration], JHEP 0612, 011 (2006) [hep-ex/0610034]. 